EDO-EP-31

August, 2000

\title{
Bosonic Fields in the String-like Defect Model
}

\author{
Ichiro Oda \\ Edogawa University, 474 Komaki, Nagareyama City, Chiba 270-0198, JAPAN
}

\begin{abstract}
We study localization of bosonic bulk fields on a string-like defect with codimension 2 in a general space-time dimension in detail. We show that in cases of spin 0 scalar and spin 1 vector fields there are an infinite number of massless Kaluza-Klein (KK) states which are degenerate with respect to the radial quantum number, but only the massless zero mode state among them is coupled to fermion on the string-like defect. It is also commented on interesting extensions of the model at hand to various directions such as 'little' superstring theory, conformal field theory and a supersymmetric construction.
\end{abstract}

\footnotetext{
${ }^{1}$ E-mail address: ioda@edogawa-u.ac.jp
} 


\section{Introduction}

In the theories where our four dimensional world is a 3-brane embedded in a higher dimensional space [1, 2, 3, 4, 5], the conventional Kaluza-Klein scenario would be modified drastically. In most works on the Kaluza-Klein compactification thus far, a higher dimensional manifold is assumed to be composed of as a direct product of a non-compact four dimensional Minkowski space-time and a compact internal manifold with the size of the compact space being set by the Planck scale. However, in this approach it seems to be quite difficult to stabilize the size of all the internal dimensions around the Planck scale via some non-perturbative effects. This problem should be solved in the brane world 4 .

In recent years, an alternative scenario of the compactification has been put forward [5]. This new idea is based on the possibility that our world is a 3-brane embedded in a higher dimensional space-time with non-factorizable warped geometry. In this scenario, we are free from the moduli stabilization problem in the sense that the internal manifold is noncompact and does not need to be compactified to the Planck scale any more, which is one of reasons why this new compactification scenario has attracted so much attention. An important ingredient of this scenario is that all the matter fields are thought of as confined to the a 3-brane, whereas gravity is free to propagate in the extra dimensions. Such localization of matters would be indeed possible in D-brane theory [6] and M-theory [7], but at present it is far from complete to realize the Rundall-Sundrum model [5] within the framework of superstring theory. Thus, it is worthwhile to explore whether such localization is also possible in the local field theory.

In fact, the localization mechanism has been recently investigated in $A d S_{5}$ space [8, 9, 10, 11, 12]. In particular, it is shown that spin 0 field is localized on a brane with positive tension which also localizes the graviton [11], while spin 1 field is not localized neither on a brane with positive tension nor on a brane with negative tension [9, 11]. Moreover, it is shown that spin $1 / 2$ and $3 / 2$ fields are localized not on a brane with positive tension but on a brane with negative tension 110, 11]. Thus, in order to fulfill the localization of Standard Model particles on a brane with positive tension, it seems that some additional interactions except gravity must be also introduced in the bulk.

More recently, the possibility of extending the Randall-Sundrum domain wall model to higher dimensional topological objects was explored [13, 14, 15, 16, 17, 18, 19, 20, 21, 22, 23. In particular, we find that Einstein's equations admit a string-like defect with codimension 2 in addition to a domain wall with codimension 1 f. In particular, the existence of the string-like defect makes it possible to think of a 3-brane in the six dimensional anti-de Sitter space.

In this string-like defect model, the localization of bulk fields has been also investigated.

\footnotetext{
${ }^{2}$ In a supersymmetric model, flat directions could appear so that the stability problem of moduli seems at first glance to be not so important as in a non-supersymmetric model. But in this case, we need the fine-tuning of the parameters.

${ }^{3}$ In this terminology, topological defects with codimension 3 and 4 , respectively, would be called a monopole-like defect and an instanton-like defect.
} 
In Ref. [18], it is shown that spin 2 graviton is localized on the 3 -brane and the corrections to Newton's law are more supressed than in the domain wall model. Afterwards, the present author has explored the localization of various spin fields on the string-like defect in a general dimension and obtained the following facts [22] : spin 0, 1 and 2 bosonic fields are localized on a string-like defect with the exponentially decreasing warp factor, whereas spin $1 / 2$ and $3 / 2$ fermionic fields are localized on a defect with the exponentially increasing warp factor. These results for the localization of various spin fields coincide with the corresponding ones [11] in the Randall-Sundrum model [0] and many brane model [24, 25] except spin 1 vector field. It is of interest that there is no localized vector field on the brane in the domain wall model f, while vector field can be localized on the defect in the string-like model. This phenomenon can be briefly explained as follows: In the Randall-Sundrum model, we can see that the overall coefficient in front of gauge field action is divergent so that we do not have a normalizable zero mode of the bulk gauge field. On the other hand, in our string-like model, we have an additional warped factor coming from part of the angular variable in the background metric in addition to the conventional warped factor. Combined with these two warped factors, the coefficient in front of the action becomes finite so the zero mode of the bulk gauge field is normalizable and is consequently localized on the string-like defect.

One aim of the present paper is to investigate this interesting property of bulk bosonic fields in the string-like defect model in more detail. The case of spin 2 graviton field has been already examined in Ref. [18, 23, so we will concentrate on the study of spin 0 scalar and spin 1 vector fields. We will show that there are an infinite number of massless Kaluza-Klein $(\mathrm{KK})$ modes which are degenerate with respect to the radial quantum number, but only one massless field among them is coupled to fermion on the string-like defect. Moreover, the KK excitations of gauge field have vanishing coupling to spin $1 / 2$ fermion on the defect, so gauge field can exist in the bulk without meeting any phenomenological constraints on the model, which should be contrasted with the Randall-Sundrum domain wall model where the strong coupling of the KK excitations of gauge field to the brane fermion gave rise to a potential internal inconsistency within the theory [8, 9].

This paper is organized as follows. In the next section, we review a string-like defect solution with codimension 2. In Section 3, the Kaluza-Klein decomposition of scalar field is studied in a background obtained in Section 2. Then, in Section 4, the procedure used in Section 3 is applied to the case of gauge field. The final section is devoted to discussions.

\section{A string-like defect}

Let us start with a brief review of a string-like defect solution to Einstein's equations with sources to fix our notations and conventions [22]. We consider Einstein's equations with a

\footnotetext{
${ }^{4}$ See Ref. 26] for an interesting possibility of electric charge non-conservation in brane world where a higher-dimensional generalization of the Randall-Sundrum model is used in order to localize gauge fields on a brane.
} 
bulk cosmological constant $\Lambda$ and an energy-momentum tensor $T_{M N}$ in general $D$ dimensions:

$$
R_{M N}-\frac{1}{2} g_{M N} R=-\Lambda g_{M N}+\kappa_{D}^{2} T_{M N},
$$

where $\kappa_{D}$ denotes the $D$-dimensional gravitational constant. Throughout this article we follow the standard conventions and notations of the textbook of Misner, Thorne and Wheeler [27].

Let us adopt the following metric ansatz:

$$
\begin{aligned}
d s^{2} & =g_{M N} d x^{M} d x^{N} \\
& =g_{\mu \nu} d x^{\mu} d x^{\nu}+\tilde{g}_{a b} d x^{a} d x^{b} \\
& =e^{-A(r)} \hat{g}_{\mu \nu} d x^{\mu} d x^{\nu}+d r^{2}+e^{-B(r)} d \Omega_{n-1}^{2},
\end{aligned}
$$

where $M, N, \ldots$ denote $D$-dimensional space-time indices, $\mu, \nu, \ldots$ do $p$-dimensional brane ones, and $a, b, \ldots$ do $n$-dimensional extra spatial ones, so the equality $D=p+n$ holds. (We assume $p \geq 4$.) And $\mathrm{d} \Omega_{n-1}^{2}$ stands for the metric on a unit $(n-1)$-sphere, which is concretely expressed in terms of the angular variables $\theta_{i}$ as

$$
d \Omega_{n-1}^{2}=d \theta_{2}^{2}+\sin ^{2} \theta_{2} d \theta_{3}^{2}+\sin ^{2} \theta_{2} \sin ^{2} \theta_{3} d \theta_{4}^{2}+\cdots+\prod_{i=2}^{n-1} \sin ^{2} \theta_{i} d \theta_{n}^{2} .
$$

Moreover, we shall take the ansatz for the energy-momentum tensor respecting the spherical symmetry:

$$
\begin{aligned}
T_{\nu}^{\mu} & =\delta_{\nu}^{\mu} t_{o}(r), \\
T_{r}^{r} & =t_{r}(r), \\
T_{\theta_{2}}^{\theta_{2}} & =T_{\theta_{3}}^{\theta_{3}}=\cdots=T_{\theta_{n}}^{\theta_{n}}=t_{\theta}(r),
\end{aligned}
$$

where $t_{i}(i=o, r, \theta)$ are functions of only the radial coordinate $r$.

With these ansatzs, after a straightforward calculation, Einstein's equations (1) reduce to

$$
\begin{gathered}
e^{A} \hat{R}-\frac{p(n-1)}{2} A^{\prime} B^{\prime}-\frac{p(p-1)}{4}\left(A^{\prime}\right)^{2}-\frac{(n-1)(n-2)}{4}\left(B^{\prime}\right)^{2} \\
+(n-1)(n-2) e^{B}-2 \Lambda+2 \kappa_{D}^{2} t_{r}=0, \\
e^{A} \hat{R}+(n-2) B^{\prime \prime}-\frac{p(n-2)}{2} A^{\prime} B^{\prime}-\frac{(n-1)(n-2)}{4}\left(B^{\prime}\right)^{2} \\
+(n-2)(n-3) e^{B}+p A^{\prime \prime}-\frac{p(p+1)}{4}\left(A^{\prime}\right)^{2}-2 \Lambda+2 \kappa_{D}^{2} t_{\theta}=0, \\
\frac{p-2}{p} e^{A} \hat{R}+(p-1)\left(A^{\prime \prime}-\frac{n-1}{2} A^{\prime} B^{\prime}\right)-\frac{p(p-1)}{4}\left(A^{\prime}\right)^{2} \\
+(n-1)\left[B^{\prime \prime}-\frac{n}{4}\left(B^{\prime}\right)^{2}+(n-2) e^{B}\right]-2 \Lambda+2 \kappa_{D}^{2} t_{o}=0,
\end{gathered}
$$


where the prime denotes the differentiation with respect to $r$, and $\hat{R}$ is the scalar curvature associated with the brane metric $\hat{g}_{\mu \nu}$. Here we define the cosmological constant on the $(p-1)$ brane, $\Lambda_{p}$, by the equation

$$
\hat{R}_{\mu \nu}-\frac{1}{2} \hat{g}_{\mu \nu} \hat{R}=-\Lambda_{p} \hat{g}_{\mu \nu}
$$

In addition, the conservation law for the energy-momentum tensor, $\nabla^{M} T_{M N}=0$ takes the form

$$
t_{r}^{\prime}=\frac{p}{2} A^{\prime}\left(t_{r}-t_{o}\right)+\frac{n-1}{2} B^{\prime}\left(t_{r}-t_{\theta}\right) .
$$

Our purpose is to find a string-like defect solution, that is, $n=2$, with a warp factor $A(r)=c r$ ( $c$ is a positive constant) to the above equations. (The case of $n=1$ corresponds to a domain wall solution.) The necessity of this exponentially decreasing warp factor is to bind gravity to the p-brane. For generality, we consider a general space-time dimension $D$ and a general brane dimension $p$ with $D=p+2$, but the physical interest, of course, lies in the case of six space-time dimensions $(D=6)$ and a 3 -brane $(p=4)$. In the case of $n=2$, under the ansatz $A(r)=c r$, Einstein equations (5), (6), (7) are of the form

$$
\begin{gathered}
e^{c r} \hat{R}-\frac{p}{2} c B^{\prime}-\frac{p(p-1)}{4} c^{2}-2 \Lambda+2 \kappa_{D}^{2} t_{r}=0, \\
e^{c r} \hat{R}-\frac{p(p+1)}{4} c^{2}-2 \Lambda+2 \kappa_{D}^{2} t_{\theta}=0, \\
\frac{p-2}{p} e^{c r} \hat{R}-\frac{p-1}{2} c B^{\prime}-\frac{p(p-1)}{4} c^{2}+B^{\prime \prime}-\frac{1}{2}\left(B^{\prime}\right)^{2}-2 \Lambda+2 \kappa_{D}^{2} t_{o}=0,
\end{gathered}
$$

and the conservation law takes the form

$$
t_{r}^{\prime}=\frac{p}{2} c\left(t_{r}-t_{o}\right)+\frac{1}{2} B^{\prime}\left(t_{r}-t_{\theta}\right) .
$$

From these equations, general solutions can be found as follows:

$$
d s^{2}=e^{-c r} \hat{g}_{\mu \nu} d x^{\mu} d x^{\nu}+d r^{2}+e^{-B(r)} d \theta^{2},
$$

where

$$
\begin{aligned}
B(r) & =c r+\frac{4}{p c} \kappa_{D}^{2} \int^{r} d r\left(t_{r}-t_{\theta}\right), \\
c^{2} & =\frac{1}{p(p+1)}\left(-8 \Lambda+8 \kappa_{D}^{2} \alpha\right), \\
\hat{R} & =\frac{2 p}{p-2} \Lambda_{p}=-2 \kappa_{D}^{2} \beta .
\end{aligned}
$$


Here $t_{\theta}$ must take a definite form, which is given by

$$
t_{\theta}=\beta e^{c r}+\alpha
$$

with $\alpha$ and $\beta$ being some constants. Moreover, in order to guarantee the positivity of $c^{2}, \alpha$ should satisfy an inequality $-8 \Lambda+8 \kappa_{D}^{2} \alpha>0$.

Two types of special solution deserve more scrutiny. A specific solution is the one without sources $\left(t_{i}=0\right)$. Then we get a special solution which was found for a local string in Ref. [18], and for a global string in Ref. [15]:

$$
d s^{2}=e^{-c r} \hat{g}_{\mu \nu} d x^{\mu} d x^{\nu}+d r^{2}+R_{0}^{2} e^{-c r} d \theta^{2},
$$

with $R_{0}$ being a length scale which we take to be of order unit. Here the positive constant $c$, the brane scalar curvature and the brane cosmological constant are respectively given by

$$
\begin{aligned}
c^{2} & =\frac{-8 \Lambda}{p(p+1)}, \\
\hat{R} & =\frac{2 p}{p-2} \Lambda_{p}=0 .
\end{aligned}
$$

In this case, as in the corresponding domain wall solution, the bulk geometry is the anti-de Sitter space, and the brane geometry is Ricci-flat with vanishing cosmological constant. It has been recently found that this special solution corresponds to a local defect in the sense that the energy-momentum tensor is strictly vanishing outside the string core [18, 23]

Another specific solution occurs when we have the spontaneous symmetry breakdown $t_{r}=-t_{\theta}$ [17]:

$$
d s^{2}=e^{-c r} \hat{g}_{\mu \nu} d x^{\mu} d x^{\nu}+d r^{2}+R_{0}^{2} e^{-c_{1} r} d \theta^{2}
$$

where

$$
\begin{aligned}
c^{2} & =\frac{1}{p(p+1)}\left(-8 \Lambda+8 \kappa_{D}^{2} t_{\theta}\right)>0 \\
c_{1} & =c-\frac{8}{p c} \kappa_{D}^{2} t_{\theta}, \\
\hat{R} & =\frac{2 p}{p-2} \Lambda_{p}=0 .
\end{aligned}
$$

Notice that this solution is more general than the previous one (18) since this solution reduces to (18) when $t_{\theta}=0$. In Ref. [23], the solution (20) was called a global defect since there appears a hedgehog type configuration outside the string core.

To close this section, let us comment on an interesting global defect recently found in a general dimension in Ref. [23]. To gain the global topological defect, the antisymmetric tensor field with rank $n-2$ is added to the Einstein-Hilbert action with a cosmological constant. 
Then the energy-momentum tensor associated with the $(n-2)$-form field in the bulk has the property

$$
t_{0}=t_{r}=-t_{\theta}
$$

The ansatz taken in Ref. 23] is

$$
A(r)=c r, B(r)=\text { constant } .
$$

With this ansatz (23), it is easy to see that Einstein's equations (5), (61), (7) and the conservation law (9) require important equations

$$
t_{0}=t_{r}=\text { constant }, t_{\theta}=\text { constant },
$$

in addition to the other inessential equations for the present consideration. These conditions (24) is more general than (22), so if an energy-momentum tensor satisfies (24), Einstein's equations with such energy-momentum tensor would admit the global topological defect with the background metric (23) as a solution in a general space-time dimension. Finally, note that this new global defect has the same property as the domain wall with respect to the localization of various bulk fields.

\section{Kaluza-Klein decomposition of scalar field}

In previous paper, it was shown that spin 0,1 , and 2 bosonic fields are localized on the pbrane defect with the exponentially decreasing warp factor, while spin $1 / 2$ and $3 / 2$ fermionic fields are not so in the string-like defect [22]. Thus, it is natural to consider first the case of a bulk scalar field. The case of a bulk vector field will be examined in the next section. The spin 2 graviton was examined in detail in Ref. [18] so we skip this case in this paper. From now on, for clarity we shall limit our attention to a local string-like solution (18) since the generalization to a global solution (20) is straightforward. Of course, we have implicitly assumed that various bulk fields considered below make little contribution to the bulk energy so that the solution (18) remains valid even in the presence of bulk fields.

Let us consider the action of a massless real scalar coupled to gravity:

$$
S_{\Phi}=-\frac{1}{2} \int d^{D} x \sqrt{-g} g^{M N} \partial_{M} \Phi \partial_{N} \Phi,
$$

from which the equation of motion can be derived:

$$
\frac{1}{\sqrt{-g}} \partial_{M}\left(\sqrt{-g} g^{M N} \partial_{N} \Phi\right)=0 .
$$

From now on we shall take $\hat{g}_{\mu \nu}=\eta_{\mu \nu}$ and define $P(r)=e^{-c r}$. In the background metric (18), the equation of motion (26) reads

$$
P^{-1} \eta^{\mu \nu} \partial_{\mu} \partial_{\nu} \Phi+P^{-\frac{p+1}{2}} \partial_{r}\left(P^{\frac{p+1}{2}} \partial_{r} \Phi\right)+\frac{1}{R_{0}^{2}} P^{-1} \partial_{\theta}^{2} \Phi=0 .
$$


Let the KK expansion of $\Phi$ be given by

$$
\Phi\left(x^{M}\right)=\sum_{n, l=0}^{\infty} \phi^{(n, l)}\left(x^{\mu}\right) \frac{\chi_{n}(r)}{\sqrt{R_{0}}} Y_{l}(\theta) .
$$

Here $Y_{l}(\theta)$ are in general the eigenfunction of the scalar Laplacian $\Delta$ on a unit $(n-1)$ sphere with the eigenvalues $l(l+n-2)$. Now we are taking account of a stringy defect with codimension 2 , i.e., $n$ is chosen to 2 , so we have an equation

$$
\Delta Y_{l}(\theta)=l^{2} Y_{l}(\theta)
$$

with $l=0,1,2, \cdots$. And $Y_{l}(\theta)$ satisfy the following orthonormality condition

$$
\int_{0}^{2 \pi} d \theta Y_{l}(\theta) Y_{l^{\prime}}(\theta)=\delta_{l l^{\prime}}
$$

Using the KK expansion (28), the equation of motion (27) reduces to the well-known Klein-Gordon's equation with the KK masses $m_{n}$ :

$$
\left(\eta^{\mu \nu} \partial_{\mu} \partial_{\nu}-m_{n}^{2}\right) \phi^{(n, l)}=0
$$

where we have required $\chi$ to satisfy the following differential equation:

$$
-\left(P^{-\frac{p-1}{2}} \partial_{r} P^{\frac{p+1}{2}} \partial_{r}-\frac{l^{2}}{R_{0}^{2}}\right) \chi_{n}=m_{n}^{2} \chi_{n} .
$$

Actually, it is easily shown that by means of Eqs. (28), (29), (30) and (32) the starting action (25) can be written as

$$
S_{\Phi}=-\frac{1}{2} \sum_{n, l=0}^{\infty} \int d^{p} x\left[\eta^{\mu \nu} \partial_{\mu} \phi^{(n, l)} \partial_{\nu} \phi^{(n, l)}+m_{n}^{2} \phi^{(n, l)} \phi^{(n, l)}\right],
$$

where we have also used the orthonormality condition

$$
\int_{0}^{\infty} d r P^{\frac{p-1}{2}} \chi_{n} \chi_{n^{\prime}}=\delta_{n n^{\prime}}
$$

To analyse the scalar KK mass spectrum, it is necessary to solve Eq. (32) explicitly. Defining $M_{n}^{2}=m_{n}^{2}-\frac{l^{2}}{R_{0}^{2}}, z_{n}=\frac{2}{c} M_{n} P^{-\frac{1}{2}}$ and $h_{n}=P^{\frac{p+1}{4}} \chi_{n}$, Eq. (32) can be written in the form

$$
\left[\frac{d^{2}}{d z_{n}^{2}}+\frac{1}{z_{n}} \frac{d}{d z_{n}}+\left\{1-\frac{1}{z_{n}^{2}}\left(\frac{p+1}{2}\right)^{2}\right\}\right] h_{n}=0
$$

which is nothing but the Bessel equation of order $\frac{p+1}{2}$. Thus, the solutions are of the form

$$
\chi_{n}=\frac{1}{N_{n}} P^{-\frac{p+1}{4}}\left[J_{\frac{p+1}{2}}\left(z_{n}\right)+\alpha_{n} Y_{\frac{p+1}{2}}\left(z_{n}\right)\right]
$$


where $N_{n}$ are the wavefunction normalization constants and $\alpha_{n}$ are constant coefficients. The differential operator in (32) is self-adjoint provided that one imposes the boundary conditions 18

$$
\chi_{n}^{\prime}(0)=\chi_{n}^{\prime}(\infty)=0
$$

These boundary conditions lead to the relations

$$
\begin{aligned}
\alpha_{n} & =-\frac{J_{\frac{p-1}{2}}\left(z_{n}(0)\right)}{Y_{\frac{p-1}{2}}\left(z_{n}(0)\right)} \\
& =-\frac{J_{\frac{p-1}{2}}\left(z_{n}(\bar{r})\right)}{Y_{\frac{p-1}{2}}\left(z_{n}(\bar{r})\right)}
\end{aligned}
$$

where $\bar{r}$ indicates the infrared cutoff, which is taken to be an infinity at the end of calculations. Incidentally, in deriving (38) we have used the formula holding in the Bessel functions

$$
Z_{\nu}^{\prime}(z)=Z_{\nu-1}(z)-\frac{\nu}{z} Z_{\nu}(z)
$$

with $Z$ being $J$ or $Y$. Now in the limit $M_{n}<<c$, the KK masses can be derived from the equation 9]

$$
J_{\frac{p-1}{2}}\left(z_{n}(\bar{r})\right)=0
$$

which gives us the approximate mass formula

$$
M_{n}=\frac{c}{2}\left(n+\frac{p}{4}-\frac{1}{2}\right) \pi e^{-\frac{1}{2} c \bar{r}}
$$

Moreover, the normalization constant $N_{n}$ takes the approximate form in the limit $M_{n}<<c$,

$$
N_{n}=\sqrt{c} \frac{z_{n}(\bar{r})}{2 M_{n}} J_{\frac{p+1}{2}}\left(z_{n}(\bar{r})\right) .
$$

Note that in the limit $\bar{r} \rightarrow \infty, M_{n}$ approach zero as in the graviton [18, which is a characteristic feature of noncompact extra dimensions. The KK masses of a scalar field are given by not $M_{n}$ but $m_{n}$, so it turns out that they approach $\frac{l^{2}}{R_{0}^{2}}$. Accordingly, only the $s$-wave $(l=0)$ mode becomes massless on the string-like defect while the other modes are massive. Here it is worth noticing that the massless $s$-wave mode is degenerate with respect to the radial quantum number $n$ since the KK masses depend on only $l$ in the limit $\bar{r} \rightarrow \infty$. Thus, we are in danger of the existence of an infinite number of massless modes on the defect, which seems to be against the phenomenology. Luckily enough, however, as will be shown below, only a unique massless mode with $n=0$ couples to fermion on the defect since the coupling constant between the remaining massless modes with $n \geq 1$ and the defect fermion vanishes in the infinite volume limit. It would be then natural to identify this massless zero mode with $n=0$ as the Higgs field in our world from the phenomenological viewpoint. 
As is shown in Ref. [22], spin 1/2 fermion is localized on a defect with the exponentially rising warp factor, so it is necessary to invoke additional interactions except gravity in a model in order to localize spin $1 / 2$ fermion on our defect, which has the exponentially decreasing warp factor. In this letter, we simply consider fermion on the string-like defect.

To see that only the massless zero mode with $n=0$ couples to fermion on the defect, it is useful to examine the Yukawa coupling whose interaction term is given by

$$
S_{\bar{\Psi} \Psi \Phi}=-g_{\Phi} \int d^{D} x \sqrt{-g} \bar{\Psi} \Psi \Phi \delta(r)
$$

The integration over the angular variable and the KK expansion (28) yield

$$
S_{\bar{\Psi} \Psi \Phi}=-g_{\Phi} \sqrt{R_{0}} \int d^{p} x \bar{\Psi} \Psi \sum_{n=0}^{\infty} \phi^{(n, 0)}(x) \chi_{n}(0) .
$$

The wavefunction for the zero mode is a constant and from the orthonormality condition (34) we have the zero mode

$$
\chi_{0}=\sqrt{\frac{c(p-1)}{2}} .
$$

For the excited KK modes $\chi_{n}(0)$ with $n \geq 1$, it is easy to evaluate $\chi_{n}(0)$ in the limit $M_{n}<<c$

$$
\chi_{n}(0)=\frac{1}{N_{n}} J_{\frac{p+1}{2}}\left(\frac{2}{c} M_{n}\right)=\sqrt{c} P^{\frac{1}{4}}(\bar{r}),
$$

where (42) was used. Moreover, defining the effective p-dimensional coupling constant as $\tilde{g}_{\Phi}=g_{\Phi} \sqrt{\frac{c(p-1)}{2} R_{0}}$, the Yukawa interaction can be expressed as

$$
S_{\bar{\Psi} \Psi \Phi}=-\tilde{g}_{\Phi} \int d^{p} x \bar{\Psi} \Psi\left[\phi^{(0,0)}(x)+\sqrt{\frac{2}{p-1}} P^{\frac{1}{4}}(\bar{r}) \sum_{n=1}^{\infty} \phi^{(n, 0)}(x)\right] .
$$

From this equation, it is obvious that the effective coupling of the excited KK modes with $n \geq 1$ to the defect fermion vanishes in the limit $\bar{r} \rightarrow \infty$ owing to the presence of $P^{\frac{1}{4}}(\bar{r})$ in front of the second term. On the other hand, the massless zero mode with $n=0$ has a coupling constant of order one. Hence, only the massless zero mode lives in the string-like defect.

\section{Kaluza-Klein decomposition of vector field}

Next we turn our attention to the case of vector field. It was shown in the Randall-Sundrum model in $A d S_{5}$ space that spin 1 vector field is not localized neither on a brane with positive tension nor on a brane with negative tension so the Dvali-Shifman mechanism 28] must be invoked for the vector field localization [11, 9]. On the other hand, we have shown that spin 
1 vector field is localized on a string-like defect like spin 0 scalar and spin 2 graviton fields 222. So we do not need to introduce additional mechanism for the vector field localization in the case at hand. The localization of vector field on the defect therefore allows us to think of the bulk vector field.

Let us start with the action of the $U(1)$ vector field:

$$
S_{A}=-\frac{1}{4} \int d^{D} x \sqrt{-g} g^{M N} g^{R S} F_{M R} F_{N S}
$$

where $F_{M N}=\partial_{M} A_{N}-\partial_{N} A_{M}$ as usual. (The extension to the case of non-Abelian gauge fields is straightforward.) From this action the equations of motion are given by

$$
\frac{1}{\sqrt{-g}} \partial_{M}\left(\sqrt{-g} g^{M N} g^{R S} F_{N S}\right)=0 .
$$

With the background metric (18) and the gauge conditions $\partial_{\mu} A^{\mu}=A_{\theta}=0$, these equations become

$$
\begin{gathered}
\left(\eta^{\mu \nu} \partial_{\mu} \partial_{\nu}+P^{\frac{3-p}{2}} \partial_{r} P^{\frac{p-1}{2}} \partial_{r}+\frac{1}{R_{0}^{2}} \partial_{\theta}^{2}\right) A_{\lambda}-P^{\frac{3-p}{2}} \partial_{r} P^{\frac{p-1}{2}} \partial_{\lambda} A_{r}=0 \\
\left(\eta^{\mu \nu} \partial_{\mu} \partial_{\nu}+\frac{1}{R_{0}^{2}} \partial_{\theta}^{2}\right) A_{r}=0 \\
\partial_{r}\left(P^{\frac{p-1}{2}} \partial_{\theta} A_{r}\right)=0 .
\end{gathered}
$$

Let us take the following forms of the KK decomposition for later convenience:

$$
\begin{aligned}
& A_{\mu}\left(x^{M}\right)=\sum_{n, l=0}^{\infty} A_{\mu}^{(n, l)}\left(x^{\mu}\right) \frac{f_{n}(r)}{\sqrt{R_{0}}} Y_{l}(\theta), \\
& A_{r}\left(x^{M}\right)=\sum_{l=0}^{\infty} A_{r}^{(l)}\left(x^{\mu}\right) \frac{g(r)}{\sqrt{R_{0}}} Y_{l}(\theta) .
\end{aligned}
$$

Then, from Eq. (51) we have

$$
\left(\eta^{\mu \nu} \partial_{\mu} \partial_{\nu}-\frac{l^{2}}{R_{0}^{2}}\right) A_{r}^{(l)}\left(x^{\mu}\right)=0 .
$$

In addition, Eq. (52) leads to a general solution for $g(r)$.

$$
g(r)=\alpha P^{-\frac{p-1}{2}}
$$

with $\alpha$ being an integration constant. Finally, using (55), Eq. (50) reduces to the form

$$
\left(\eta^{\mu \nu} \partial_{\mu} \partial_{\nu}-m_{n}^{2}\right) A_{\lambda}^{(n, l)}\left(x^{\mu}\right)=0
$$


Here we have required $f_{n}(r)$ to satisfy the differential equation

$$
-\left(P^{-\frac{p-3}{2}} \partial_{r} P^{\frac{p-1}{2}} \partial_{r}-\frac{l^{2}}{R_{0}^{2}}\right) f_{n}(r)=m_{n}^{2} f_{n}(r) .
$$

As in the case of a scalar field, let us substitute the KK expansion (53) and the solution (55) into the starting action (48), whose result is given by

$$
\begin{array}{r}
S_{A}=\int d^{p} x \sum_{n, l=0}^{\infty}\left[-\frac{1}{4} \eta^{\mu \nu} \eta^{\lambda \rho} F_{\mu \lambda}^{(n, l)} F_{\nu \rho}^{(n, l)}-\frac{1}{2} m_{n}^{2} \eta^{\mu \nu} A_{\mu}^{(n, l)} A_{\nu}^{(n, l)}\right] \\
-\frac{\alpha^{2}}{c(p-1)}\left[P(\bar{r})^{-\frac{p-1}{2}}-1\right] \times \int d^{p} x \sum_{l=0}^{\infty}\left[\eta^{\mu \nu} \partial_{\mu} A_{r}^{(l)} \partial_{\nu} A_{r}^{(l)}+\frac{l^{2}}{R_{0}^{2}} A_{r}^{(l)} A_{r}^{(l)}\right],
\end{array}
$$

where we have used (30) and the orthonormality condition for $f_{n}(r)$

$$
\int_{0}^{\infty} d r P^{\frac{p-3}{2}} f_{n} f_{n^{\prime}}=\delta_{n n^{\prime}}
$$

Note that the coefficient in front of the action of the scalar field $A_{r}$ becomes divergent in the limit $\bar{r} \rightarrow \infty$, but this divergence can be absorbed in the redefinition of the field $A_{r}^{(l)}$. That is, by performing the field-redefinition $A_{r}^{(l)} \rightarrow \alpha \sqrt{\frac{2}{c(p-1)}} \sqrt{P(\bar{r})^{-\frac{p-1}{2}}-1} A_{r}^{(l)}$, we arrive at the expression

$$
\begin{aligned}
S_{A}=\int d^{p} x \sum_{n, l=0}^{\infty} & {\left[-\frac{1}{4} \eta^{\mu \nu} \eta^{\lambda \rho} F_{\mu \lambda}^{(n, l)} F_{\nu \rho}^{(n, l)}-\frac{1}{2} m_{n}^{2} \eta^{\mu \nu} A_{\mu}^{(n, l)} A_{\nu}^{(n, l)}\right] } \\
& -\frac{1}{2} \int d^{p} x \sum_{l=0}^{\infty}\left[\eta^{\mu \nu} \partial_{\mu} A_{r}^{(l)} \partial_{\nu} A_{r}^{(l)}+\frac{l^{2}}{R_{0}^{2}} A_{r}^{(l)} A_{r}^{(l)}\right] .
\end{aligned}
$$

Here notice that the second integral describes that the 'gauge-scalar' has the same structure as the action of scalar field (33). Thus, following the similar argument to the case of a scalar field, it is straightforward to show that only the massless zero mode of the 'gauge-scalar' couples to fermion on the defect. Therefore, in what follows, we shall consider the $p$-dimensional gauge field $A_{\mu}$.

To examine the KK spectrum, we can follow a similar path of argument to that of a scalar field in Section 3. This time, by defining $h_{n}=P^{\frac{p-1}{4}} f_{n}$, Eq. (57) can be written as

$$
\left[\frac{d^{2}}{d z_{n}^{2}}+\frac{1}{z_{n}} \frac{d}{d z_{n}}+\left\{1-\frac{1}{z_{n}^{2}}\left(\frac{p-1}{2}\right)^{2}\right\}\right] h_{n}=0
$$

whose solution is also expressed in terms of the Bessel functions of order $\frac{p-1}{2}$

$$
f_{n}\left(z_{n}\right)=\frac{1}{N_{n}} P^{-\frac{p-1}{4}}\left[J_{\frac{p-1}{2}}\left(z_{n}\right)+\alpha_{n} Y_{\frac{p-1}{2}}\left(z_{n}\right)\right]
$$


where $N_{n}$ are new wavefunction normalization constants and $\alpha_{n}$ are new constant coefficients. The same boundary conditions (37) for $f_{n}(r)$ lead to the relations

$$
\begin{aligned}
\alpha_{n} & =-\frac{J_{\frac{p-3}{2}}\left(z_{n}(0)\right)}{Y_{\frac{p-3}{2}}\left(z_{n}(0)\right)} \\
& =-\frac{J_{\frac{p-3}{2}}\left(z_{n}(\bar{r})\right)}{Y_{\frac{p-3}{2}}\left(z_{n}(\bar{r})\right)} .
\end{aligned}
$$

In the limit $M_{n}<<c$, the KK masses can be derived from the equation

$$
J_{\frac{p-3}{2}}\left(z_{n}(\bar{r})\right)=0
$$

which gives us the approximate mass formula

$$
M_{n}=\frac{c}{2}\left(n+\frac{p}{4}-1\right) \pi e^{-\frac{1}{2} c \bar{r}}
$$

And the normalization constant $N_{n}$ takes the approximate form

$$
N_{n}=\sqrt{c} \frac{z_{n}(\bar{r})}{2 M_{n}} J_{\frac{p-1}{2}}\left(z_{n}(\bar{r})\right) .
$$

Note that in the limit $\bar{r} \rightarrow \infty$, the KK masses of vector field are given by $\frac{l^{2}}{R_{0}^{2}}$ like the scalar case. Hence as expected, only the $s$-wave $(l=0)$ becomes massless on the p-brane defect while the other modes are massive. This time, compared to the scalar case, it is more important to show that only one massless mode with $n=0$ lives in the defect since such a massless zero mode would be regarded as the unique 'photon' on the defect.

We are now ready to consider the coupling of the gauge KK modes to spin $1 / 2$ fermion on the p-brane defect. The fermion kinetic and gauge interaction terms are given by

$$
S_{\Psi}=\int d^{D} x \sqrt{-g} \bar{\Psi} i \Gamma^{M}\left(\partial_{\mu}+i g_{A} A_{\mu}\right) \Psi \delta_{M}^{\mu} \delta(r)
$$

where the curved gamma matrices $\Gamma^{\mu}$ and the flat gamma ones $\gamma^{\mu}$ are related through the relations $\Gamma^{\mu}=P^{-\frac{1}{2}} \gamma^{\mu}$. Upon integrating over $\theta$ and using the KK expansion (53), we obtain for the gauge-fermion interaction term

$$
S_{\bar{\Psi} \Psi A}=-g_{A} \sqrt{R_{0}} \int d^{p} x \bar{\Psi} \gamma^{\mu} \sum_{n=0}^{\infty} A_{\mu}^{(n, 0)}(x) f_{n}(0) \Psi .
$$

The wavefunction for the zero mode is again a constant and the orthonormality condition (59) gives us the zero mode

$$
f_{0}=\sqrt{\frac{c(p-3)}{2}}
$$


Recall that this zero mode is localized on the string-like defect and is identified with the usual 'photon' of the p-dimensional Minkowski space-time [22] while it is not localized on the domain wall [9, 11]. For the excited KK modes $f_{n}(0)$ with $n \geq 1$, it is easy to evaluate $f_{n}(0)$ in the limit $M_{n}<<c$

$$
f_{n}(0)=\frac{1}{N_{n}} J_{\frac{p-1}{2}}\left(\frac{2}{c} M_{n}\right)=\sqrt{c} P^{\frac{1}{4}}(\bar{r}),
$$

where (66) was used. Then, defining the effective p-dimensional $U(1)$ coupling constant as $\tilde{g}_{A}=g_{A} \sqrt{\frac{c(p-3)}{2} R_{0}}$, the interaction term reads

$$
S_{\Psi}^{i n t}=-\tilde{g}_{A} \int d^{p} x \bar{\Psi} \gamma^{\mu}\left[A_{\mu}^{(0,0)}(x)+\sqrt{\frac{2}{p-3}} P^{\frac{1}{4}}(\bar{r}) \sum_{n=1}^{\infty} A_{\mu}^{(n, 0)}(x)\right] \Psi .
$$

From this equation, it is obvious that the coupling of the excited KK modes to the defect fermion vanishes in the limit $\bar{r} \rightarrow \infty$ owing to the presence of $P^{\frac{1}{4}}(\bar{r})$ as in the scalar field. On the other hand, the massless zero mode has a coupling constant of order one as desired. Thus, this model is consistent with gauge fields existing in the bulk, which should be contrasted with the Randall-Sundrum model [8, 9]. Of course, further studies are necessary to assure the consistency of the model at hand at the quantum level.

\section{Discussions}

In this paper we have explored the possibility of placing spin 0 scalar field and spin 1 vector gauge field in the bulk in the string-like defect model in detail. We have derived the scalar and the gauge field KK spectra from examination of the action of the theory and also analyzing the equations of motion.

We then computed the scalar-fermion and the gauge-fermion interactions on the stringlike defect and found that the excited KK states with respect to the radial quantum number do not couple to fermion on the defect in the infinite cutoff limit, whereas the massless zero modes, which are nothing but the 'Higgs' particle and the usual 'photon' of the Minkowski space-time in the cases of scalar and gauge boson, respectively, couple to fermion with order unit. Since it has been already shown that spin 2 graviton is localized on the defect [22] and yields the desired Newton's law with tiny correction terms on the defect [18], the model which we consider equips with desirable physical properties. Of course, to make the model at hand more realistic we need additional interactions except gravity for localizing fermions on the defect, but we wish to insist that a supergravity model corresponding to the present model would resolve this problem in a natural way even if we could localize fermionic fields by introducing additional interaction terms between bosons and fermions by hand.

Let us restrict the following argument to the case of $p=4$ and $D=6$. In this case, it is of interest to imagine that 10D superstring theory might be compactified on Calabi-Yau two-fold, i.e., K3 by the conventional Kaluza-Klein mechanism, yielding 6D theory and then 
the $6 \mathrm{D}$ theory is compactified to our four dimensional space-time according to the alternative compactification scenario discussed in this paper. Here it is worthwhile to mention that the 6D local field theory is a very interesting field theory. (Correspondingly, 6D supergravity theory possesses a richer structure than 5D supergravity theory in many respects.) For instance, the 6D local field theory is a free theory with trivial cubic scalar self-interaction with unrenormalizable Einstein-Hilbert and Yang-Mills actions, so it is expected that 'little' superstring theory may play an essential role. Furthermore, our model in 6 space-time dimensions has a physical setting where our world is a 3-brane embedded in 6D space-time with non-factorizable warped geometry. Interestingly enough, the metric of two internal dimensions is conformally flat, so the powerful (Euclidean) conformal field theory technique can be naturally applied to our model.

Finally let us comment on a supersymmetric realization of the present model. A lot of attention has been recently devoted to the construction of a supersymmetric RandallSundrum model [29, 30, 31, 32, 33, 34, 35] and advocated some no-go theorems. Then it is of interest to ask whether there are supersymmetric relations between our 6D model and 5D Randall-Sundrum model. Through a simple KK dimensional reduction, it seems that the background metric in our model reduces to the one in the Randall-Sundrum model. At the same time, $\mathrm{N}=2,6 \mathrm{D}$ supergravity would reduce to $\mathrm{N}=2,5 \mathrm{D}$ supergravity. Thus, if we cannot construct a supersymmetric version of the Randall-Sundrum model, it might be also difficult to construct a supersymmetric model of our 6D theory. But there recently appeared an interesting construction of a supersymmetric Randall-Sundrum model [35]. The corresponding construction of our 6D model is now in an active investigation so we hope to report this construction in near future.

\section{Acknowledgement}

We are indebted to M. Tonin for valuable discussions and continuous encouragement. We wish to thank Dipartimento Di Fisica, "Galileo Galilei", Universita Degli Studi Di Padova, for a kind hospitality, where most of parts of this work have been done.

\section{References}

[1] V.A. Rubakov and M.E. Shaposhnikov, Phys.Lett.B125 (1983) 136.

[2] K. Akama, in Proceeding of the Symposium on Gauge Theory and Gravitation, Nara, Japan, eds. K. Kikkawa, N. Nakanishi, and H. Nariai, Springer-Verlag, 1983, hepth/0001113. 
[3] M. Visser, Phys.Lett.B159 (1985) 22.

[4] N. Arkani-Hamed, S. Dimopoulos and G. Dvali, Phys.Lett.B429 (1998) 263, hepph/9803315.

[5] L. Randall and R. Sundrum, Phys.Rev.Lett.83 (1999) 4690, hep-th/9906064.

[6] J. Polchinski, "Tasi Lectures on D-branes", hep-th/9611050.

[7] P. Horava and E. Witten, Nucl.Phys.B460 (1996) 506, hep-th/9510209; Nucl.Phys.B475 (1996) 94, hep-th/9603142.

[8] H. Davoudias, J.L. Hewett and T.G. Rizzo, Phys.Lett.B473 (2000) 43, hep-ph/9911262.

[9] A. Pomarol, hep-ph/9911294.

[10] Y. Grossman and M. Neubert, Phys.Lett.B474 (2000) 361, hep-ph/9912408.

[11] B. Bajc and G. Gabadadze, Phys.Lett.B474 (2000) 282, hep-th/9912232.

[12] S. Chang, J. Hisano, H. Nakano, N. Okada and M. Yamaguchi, hep-ph/9912498.

[13] A. Chodos and E. Poppitz, Phys.Lett.B471 (1999) 119, hep-th/9909199.

[14] A.G. Cohen and D.B. Kaplan, Phys.Lett.B470 (1999) 52, hep-th/9910132.

[15] R. Gregory, Phys.Rev.Lett.84 (2000) 2564, hep-th/9911015.

[16] C. Csaki, J. Erlich, T.J. Hollowood and Y. Shirman, hep-th/0001033.

[17] I. Olasagasti and A. Vilenkin, hep-th/0003300.

[18] T. Gherghetta and M. Shaposhnikov, hep-th/0004014.

[19] P. Berglund, T. Hubsch and D. Minic, hep-th/0005162.

[20] M. Chaichian, M. Gogberashvili, and A.B. Kobakhidze, hep-th/0005167.

[21] A. Chodos, E. Poppitz and D. Tsimpis, hep-th/0006093.

[22] I. Oda, hep-th/0006203.

[23] T. Gherghetta, E. Roessl and M. Shaposhnikov, hep-th/0006251.

[24] I. Oda, Phys.Lett.B480 (2000) 305, hep-th/9908104.

[25] I. Oda, Phys.Lett.B472 (2000) 59, hep-th/9909048.

[26] S.L. Dubovsky, V.A. Rubakov and P.G. Tinyakov, hep-ph/0007179. 
[27] C.W. Misner, K.S. Thorne and J.A. Wheeler, Gravitation, San Francisco: Freeman (1973).

[28] G. Dvali and M. Shifman, Phys.Lett.B396 (1997) 64, hep-th/9612128.

[29] R. Kallosh and A. Linde, JHEP0002 (2000) 005, hep-th/0001071.

[30] K. Behrndt and M. Cvetic, hep-th/0001159.

[31] R. Altendorfer, J. Bagger and D. Nemeschansky, hep-th/0003117.

[32] S.P. de Alwis, A.T. Flournoy and N. Irges, hep-th/0004125.

[33] J. Maldacena and C. Nunez, hep-th/0007018.

[34] E. Bergshoeff, R. Kallosh and A. Van Proeyen, hep-th/0007044.

[35] M.J. Duff, J.T. Liu and K.S. Stelle, hep-th/0007120. 